\title{
Resource Management of Disruption Tolerant Networks
}

\author{
Iyad Tumar and Jürgen Schönwälder \\ Computer Science, Jacobs University Bremen, Germany \\ $\{$ i.tumar, j.schoenwaelder $\}$ @jacobs-university.de
}

\begin{abstract}
Disruption tolerant networks (DTNs) differ from traditional networks due to their special characteristics such as frequent partitions, intermittent connectivity, and message delivery delay. This paper presents a new idea for the resource management of sparse disruption tolerant networks where the density of nodes is insufficient to support direct end-to-end communications. The idea combines an asynchronous approach, where nodes work on their own wake up schedules without synchronized clocks, with an on-demand scheme, where a low power radio (LPR) is used to search about contacts and a high power radio (HPR) undertakes the actual data transmission.
\end{abstract}

Keywords: Disruption Tolerant Networks, Power Management.

\section{Introduction}

Disruption tolerant networks (DTNs) is a research area aiming at developing network communication when connectivity is intermittent and prone to disruptions. In general, DTNs are applicable in remote and hazardous areas where the energy sources are constrained. They are also assumed to operate over a long period of time. Therefore, several research efforts have been started to develop power management schemes for disruption tolerant networks.

In DTNs, nodes need to discover neighbors to establish communication. Searching for neighbors in sparse DTNs can consume a large amount of power compared to the power consumed by infrequent data transfers. Banerjee et al. [1] show that using an 802.11 radio to search for contacts consumes $99.5 \%$ of the total energy. Therefore, novel power management schemes are needed to address this problem and to save energy in the neighbor searching mode. Designing such power management schemes is challenging because nodes need to know when to sleep to save power and when to wake up to search for neighbors. Ideally, power management schemes should not reduce network connectivity opportunities, which would negatively affect the overall performance of the network.

There are three categories for wake-up based power management approaches. The first category are scheduled rendezvous, in which sleeping nodes wake up at the same time based on deterministic wake-up patterns (assuming synchronized nodes). The second category are asynchronous approaches with overlapping active periods within a specific number of sleep cycles for any two neighbor nodes.

D. Hausheer and J. Schönwälder (Eds.): AIMS 2008, LNCS 5127, pp. 200 204, 2008.

(C) IFIP International Federation for Information Processing 2008 
The third category are on-demand approaches where nodes can be awakened on-demand at any point of time. For example, an additional low power radio can be used to trigger a high power radio.

A new power management scheme is developed in this proposal. This scheme tries to save energy in searching mode with a minimum degradation of the overall network performance. It is based on the context aware power management (CAPM) scheme [2], and it also uses an additional low power radio interface (LPR) [3]. The rest of this paper is structured as follows: Section 2 details the research questions and outlines the proposed power management scheme and how it is being evaluated. Related work is discussed in Section 3 before we conclude the paper in Section 4.

\section{Research Questions and Proposed Approach}

The goal of this work is to develop a new power management scheme for sparse disruption tolerant networks that saves energy and extends the network life time with minimum reduction of the network connectivity opportunities. The specific research questions are:

1. What are efficient approaches to save energy in sparse DTNs?

2. Can we design power management schemes without affecting the network performance?

3. More precisely what are efficient approaches to save the maximum amount of energy in sparse DTNs with the minimum degradation of network performance?

4. What is the impact of traffic load and node speed on power management scheme in DTNs?

Our approach combines the on-demand scheme and the asynchronous scheme. It uses an additional low power radio to search about contacts and it allows each node to work on its own wake up schedule without synchronized clocks. Each node uses a fixed duty cycle. Within this duty cycle, each node wakes up for a fixed or adaptive period and sleeps for the rest of the time. When a node wakes up, it broadcasts a beacon that includes its identifier and the time it will stay active. If this node has data to deliver, it will piggyback a notification delivery (destination identifier) to the beacon. We assume that there will be overlapping active periods between nodes within a certain number of duty cycles.

We do not consider the energy consumption of computations; we only consider the energy consumption of wireless interfaces in searching mode when nodes search about each other to communicate. Two alternative operations are considered in this scheme: neighbor discovery in which a node wakes up to search about neighbor nodes for data forwarding, and data delivery in which data is exchanged among nodes. Data delivery uses the HPR to transfer the data, while neighbor discovery is based on both radios to find contacts.

There are two scenarios when using LPR and HPR radios to search about contacts. The first one is based on the LPR waking up once each duty cycle to 


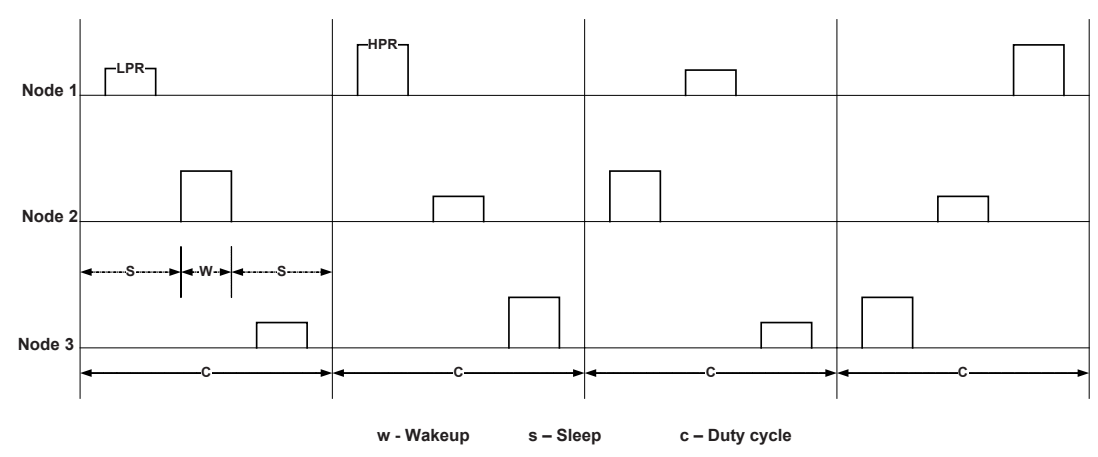

Fig. 1. Low and high power radios random wake up

search about contacts and uses the HPR on demand to transfer data after a communication link between two nodes has been established. The second scenario alters between LPR and HPR to search about contacts. In this scenario, the LPR wakes up once each two duty cycles, and the HPR wakes up each two duty cycles or when it is triggered by the LPR to undertake the data transmission after a connection is established between two nodes (see Fig. 1). Using an additional low power radio in an asynchronous manner to search about contacts leads to overlapping periods between HPR and LPR. In order to enable both radios to communicate with each other, we assume that both radios use the same MAC layer and the same frequency (only one radio will be active at any point of time). By allowing both radios to communicate with each other and by utilizing both radios to search about contacts, we expect that a significant amount of power can be saved in searching mode.

The proposed power management scheme will be implemented and evaluated by simulation. In order to evaluate our power management scheme, two DTN routing protocols will be implemented (Epidemic [4], PRoPHET [5]). We will first evaluate the performance of Epidemic and PRoPHET in the absence of power management based on the delivery ratio and the delivery delay. Then, we will investigate the impact of our power management scheme on the delivery ratio and the delivery delay for both routing schemes under different traffic loads, and node movement speeds. The following metrics will be considered:

- The Delivery Ratio is the amount of successfully received data over the total amount of delivered data.

- The Delivery Delay is the average delay per delivered message.

- The Energy Cost is the total energy consumption over the number of delivered messages (the average energy consumption to deliver a message).

- The Normalized Energy Consumption is the ratio of the energy consumption with power management over the energy consumption without power management.

We will use the network scenario that has already been used to evaluate CAPM [2]. This scenario consists of 40 nodes that are distributed over a square 
of size $1 \mathrm{~km} \times 1 \mathrm{~km}$. These nodes move according to the random waypoint model with a maximum speed of $5 \mathrm{~m} / \mathrm{s}$ and a pause time in each waypoint of 10 seconds. The communication range for $\mathrm{HPR}$ is $250 \mathrm{~m}$ with data rate $2 \mathrm{Mbps}$, while the communication range for LPR is $100 \mathrm{~m}$ with data rate $76.8 \mathrm{Kbps}$. To be sure that simulation results are correct, they will be compared with published results in [2].

\section{Related Work}

Several power management schemes have been proposed for ad hoc networks. These schemes allow nodes to disable their radios when they are not used to save energy and to prolong the network life time while keeping network connectivity $[6,7]$. Unlike our work, these schemes assume that a node has another node within its communication range most of time.

There have been several efforts to develop energy efficient medium access control (MAC) protocols $[8,9]$. These efforts are motivated by the observation that transmissions of multiple nodes in a wireless network may interfere with each other. To avoid this interference, only two nodes can communicate with each other at any point in time. Therefore, significant energy can be saved if a node sleeps while others communicate, thereby in many cases improving overall network performance. These MAC protocols propose mechanisms to increase sleeping time based on the traffic in the neighborhood. However, these MAC protocols are designed for dense networks rather than sparse networks.

There are three approaches for power management of sparse disruption tolerant networks. These approaches focus on saving energy in searching mode when nodes search about each other to communicate. The first approach presented by Jun et al. [10] assumes synchronized clocks and allows nodes to be in one of three modes, dormant (sleep) mode, search mode, and contact mode based on knowledge of future contacts. There are three levels of knowledge: (i) complete knowledge in which nodes know everything about their future contacts so they know exactly when to wake up and when to sleep, (ii) zero knowledge in which nodes have no information about each other and so they need to search about each others, (iii) partial knowledge in which nodes wake up and sleep based on probability metrics derived from statistical information. The second approach described by Jun et al. [3] tries to minimize the power consumed in searching mode by using an additional low power radio to discover contacts and to awake the high power radio to undertake the data transmission. The third approach introduced by Chuah et al. [2] is an asynchronous mechanism in which each node works on its own wake-up schedule independently. It has a fixed duty cycle which consists of a wake-up and a sleep period. Each node wakes up for a fixed or adaptive period and sleeps for the remaining time.

\section{Conclusion}

This paper presents a new power management scheme for sparse disruption tolerant networks (DTNs) where the density of nodes is insufficient to support direct 
end-to-end communication. We formulate the research questions, outline a novel power management scheme approach combining on-demand and asynchronous schemes, and we describe how this new scheme will be evaluated.

\section{Acknowledgement}

The work reported in this paper is supported by the EC IST-EMANICS Network of Excellence (\#26854).

\section{References}

1. Banerjee, N., Corner, M., Levine, B.: An Energy-Efficient Architecture for DTN Throwboxes. In: Proceeding of IEEE INFOCOM 2007, Anchorage, Alaska, USA, May 6-12 (2007)

2. Chuah, M., Xi, Y., Chang, K.: Performance Evaluation of a Power Management Scheme for DTNs. In: Proceedings of IEEE QShine, Vancouver, British Columbia, Canada, August 14-17 (2007)

3. Jun, H., Ammar, M.H., Corner, M.D., Zegura, E.W.: Hierarchical Power Management in Disruption Tolerant Networks with Traffic-Aware Optimization. In: Proceedings of the 2006 SIGCOMM Workshop on Challenged Networks (CHANTS 2006), pp. 245-252. ACM Press, New York (2006)

4. Vahdat, A., Becker, D.: Epidemic Routing for Partially Connected Ad Hoc Networks. Technical Report CS-200006, Duke University (April 2000)

5. Lindgren, A., Doria, A., Schelen, O.: Probabilistic routing in intermittently connected networks, pp. 239-254 (August 2004)

6. Tseng, Y.C., Hsu, C.S., Hsieh, T.Y.: Power-Saving Protocols for IEEE 802.11Based Multi-Hop Ad Hoc Networks. In: Proceedings of the Twenty-First Annual Joint Conference of the IEEE Computer and Communications Societies (INFOCOM 2002), June 23-27, 2002, vol. 1, pp. 200-209 (2002)

7. Zheng, R., Hou, J.C., Sha, L.: Asynchronous Wakeup for Ad Hoc Networks. In: Proceedings of the 4th ACM International Symposium on Mobile Ad Hoc Networking and Computing (MobiHoc 2003), pp. 35-45. ACM Press, New York (2003)

8. Singh, S., Raghavendra, C.S.: PAMAS: Power Aware Multi-Access protocol with Signalling for Ad Hoc Networks. ACM Computer Communication Review 28(3), 5-26 (1998)

9. Ye, W., Heidemann, J., Estrin, D.: An Energy-Efficient MAC protocol for Wireless Sensor Networks. In: Proceedings of the IEEE INFOCOM, New York, NY, USA, June 2002, pp. 1567-1576 (2002)

10. Jun, H., Ammar, M., Zegura, E.: Power Management in Delay Tolerant Networks: A Framework and Knowledge-Based Mechanisms. In: IEEE SECON 2005 Second Annual IEEE Communications Society Conference on Sensor and Ad Hoc Communications and Networks, pp. 418-429 (September 2005) 